\title{
Chiral Stationary Phases and their Relationship with Enantiomer Structures in Enantioseparation Research of Analytical Laboratory
}

\author{
Zhi-jian He, ${ }^{1,2}$ Hang Song, ${ }^{1}$ Yi-wen Zhang, ${ }^{1}$ Dao-cai Wang, ${ }^{1}$ and Shun Yao ${ }^{1, *}$ \\ 1 School of Chemical Engineering, Sichuan University, Chengdu 610065, P.R. China. cusack@scu.edu.cn \\ 2 School of Chemistry and Chemical Engineering, Mianyang Normal University, Mianyang 621000, P. R. China
}

Received March 12 ${ }^{\text {th }}, 2014$; Accepted November $13^{\text {th }}, 2014$

\begin{abstract}
Chiral stationary phases (CSPs) and molecular structure of enantiomers are two independent but related aspects in enantioseparation, which are discussed on the basis of the experimental data from the previous study. Two enantioseparation experiments are performed to illustrate the relationship between enantiomer structures and chiral stationary phases, one is the resolution of mandelic acid derivatives and the other is about prasugrel. Thermodynamic mechanism and theoretical study with computational chemistry method is helpful to understand the interactions of enantiomer and CSPs.
\end{abstract}

Key words: Enantioseparation; Enantiomer Structures; CSPs; Mandelic Acid; Prasugrel.

\section{Introduction}

When liquid chromatography is used to separate enantiomers, it is possible the expectation of researchers can be realized by the existing hundreds of commercial chiral columns. The choice of a suitable chromatographic system usually requires researchers to carry out a lot of experimental work, which actually is on the basis of rich experimental experience, comprehensive consideration and reasonable analysis for chiral resolution process [1]. If target enantiomers could not be separated in a few available CSPs, the derivatization reagent could be used to modify their structures through simple and reversible procedure until the complete separation can be obtained. For instance, on the column packed with triacetate cellulose stationary phase (e.g. Chiralcel OD-H column of Daicel Chemical Industries Ltd., Tokyo, Japan), it was found the pre-column derivatization through the introduction of the nitrobenzene and chlorobenzene para groups into the enantiomers structure (e.g. $\alpha$-bromobutyric acid) can obviously improve their resolution [2], and the simple derivatization reactions (esterification or salt forming) could realize the successful separation of a large amount of racemates.

Besides the structures of enantiomers, the type of chiral stationary phase (CSP) is another important factor in those successful resolutions. It is a universally acknowledged that, chiral stationary phases can be divided into two different immobilized ways for chiral selector, which are bonded and coated way according to various fixed methods on solid support. In the comparison of these two, the coated way has better solvent tolerance and broader choice scope for mobile phase, so it gradually becomes a new generation of chiral stationary phase in rapid development [3]. On the other hand, several new bonded chiral stationary phases such as polysaccharide, cyclodextrin, protein,
Resumen. Fases estacionarias quirales (CSP) y estructura molecular de enantiómeros son dos aspectos relacionados entre sí, pero independientes en la separación cromatográfica de estos compuestos; ambos aspectos se discuten en este trabajo con base en los datos experimentales de los estudios anteriores. Se llevaron a cabo dos experimentos para ilustrar la relación entre las estructuras de enantiómeros y fases estacionarias quirales; uno con los derivados del ácido mandélico y el otro con prasugrel. Son útiles los mecanismo termodinámico y el estudio teórico mediante herramientas de la química computacional para entender las interacciones de enantiómero con CCSps.

Palabras clave: Separación enantiomérica; estructura de enantiómeros; estructuras enantiómericas; CSPs; ácido mandelic; prasugrel.

macrocyclic antibiotics, crown ethers for HPLC analysis also have been applied in recent years [4].

The characteristics and applicable scope of several available CSPs in our and many laboratories are shown in Table 1, which are the most representative commercial CSPs.

Among them brush-type CSPs were invented by Pirkle's group since the early 1980s [5], and this type of CSPs (such as DNB-Leu, DNB-PG, Whelk-O1, Whelk-O2, ULMO, $\alpha$-Burke, $\beta$-Gem 1, all from Regis Technologies, Inc. USA) have been extensively used for chiral analysis. Table 2 summarizes the successful separation results with various CSPs mainly in our daily analytical work. Every pair of enantiomers has been tried by all of above CSPs and the most suitable CSPs are explored. By comparison of the results in Table 2, it is found cellulose type Chiralcel OD-H (in coated way) could effectively separate the most chiral targets, and the second is "brush" type WhelkO1 (in bonded way). Above facts support the fact from one aspect that polysaccharide and its derivatives have become one of the most widely used chiral stationary phases. At present the commercial polysaccharide derivative CSPs have many varieties well known for good versatility and durability, such as Chiralpak IA, IB and IC (from Daicel Co., Ltd., Japan). Their performance is different and dependent on the backbone as well as on the side chain of the polymer. Facing so many CSPs, even experienced chromatographers may find the choice of column along with the most appropriate mobile phase to be problematic and a bit overwhelming. Here it is suggested to select polysaccharide derivative CSPs firstly, then "brush" type, protein-based and ligand-exchange type successively.

In the following content, this paper focused on two independent but related aspects (type of chiral stationary phases and molecular structure of enantiomers) on the basis of separation experiments to discuss separation rules and related inherent 
Table 1. Characteristics and application of some popular CSPs.

\begin{tabular}{|c|c|c|c|c|}
\hline CSP type & System & $\begin{array}{l}\text { Fixed ways of } \\
\text { chiral selector }\end{array}$ & Structural characteristics & Scope of application \\
\hline Whelk-O1 & $\begin{array}{l}\text { Normal } \\
\text { phase }\end{array}$ & Bonded on silica & $\begin{array}{l}\text { Brush type with amide chiral selector } \\
\text { (naproxen) }\end{array}$ & $\begin{array}{l}\text { Application scope is broad, including amide, } \\
\text { epoxide, esters, urea, carbamate compounds, } \\
\text { ether, ring ethyleneimine compounds, phosphate, } \\
\text { aldehyde, ketone, carboxylic acid, alcohol and } \\
\text { NSAIDs }\end{array}$ \\
\hline $\begin{array}{l}\text { Chiralcel } \\
\text { OD-H }\end{array}$ & $\begin{array}{l}\text { Normal } \\
\text { phase }\end{array}$ & Coated on silica & Cellulose carbamate derivatives & $\begin{array}{l}\text { Enantiomers with amide, aromatic, carbonyl, nitro, } \\
\text { sulfonyl, cyanogroup, hydroxyl and amine groups } \\
\text { and carboxylic acid. Especially suitable for } \beta \text { - } \\
\text { blockers steroids }\end{array}$ \\
\hline $\begin{array}{l}\text { Chirex3126 } \\
\text { (D)- } \\
\text { penicillamine }\end{array}$ & $\begin{array}{l}\text { Reversed } \\
\text { phase }\end{array}$ & Bonded on silica & $\begin{array}{l}\text { Ligand exchange type with a derivative } \\
\text { of D-penicillamine as chiral selector }\end{array}$ & $\begin{array}{l}\text { Alpha amino acid and its derivatives, alpha } \\
\text { hydroxy acid, amino alcohols }\end{array}$ \\
\hline
\end{tabular}

Table 2. Seventeen racemic compounds and their most suitable CSPs.

\begin{tabular}{llc}
\hline CSP type & Racemic compounds of enantioseparation & $\begin{array}{c}\text { The percentage of successfully separated } \\
\text { compounds in total listed samples }\end{array}$ \\
\hline Whelk-O1 & $\begin{array}{l}\text { benzene propyl alcohol [6], naproxen [7], ibuprofen [8], karen } \\
\text { crowe [9] }\end{array}$ & $23.5 \%$ \\
DNB-PG & procaterol [10], phenylethylamine [11] & $11.8 \%$ \\
Chiralcel OD-H & $\gamma$ - lactam [12], 2- Bromide butyric acid [13], 3-tert-butyl & $47.1 \%$ \\
& adipic acid [14], acetyl four hydrogen thiazole -2- sulfur \\
& ketone-4-carboxylic acid [15], sec-butylamine [16], 2- \\
& chloropropionic acid [17], thiazolidine-2-carboxylic acid, & \\
& trans-3-oxygen mixed tricyclic (2.2.1.0) heptanoic -7- & $11.8 \%$ \\
Kromasil CHI-DMB & carboxylic acid [18] & $5.8 \%$ \\
Chirex 3126 (D)- penicillamine & panthenol [19], 2- aminomethyl piperidine [20] & \\
\hline
\end{tabular}

interaction mechanism. Two experiments have been performed to illustrate the relationship between enantiomer structures and chiral stationary phases, one is the enantioseparation of mandelic acid derivatives and the other is about prasugrel. The study is aimed to summarize more rules for the study of enantiomers resolution mechanism.

\section{Experimental}

\section{Chemicals and Reagents}

(Rac)-mandelic acid and prasugrel (above 98\% of chemical purity) were purchased from Alfa Aesar (Tianjin, China). Hex- ane and ethanol of HPLC grade were supplied by Hangjia Chemical Co., Ltd. (Chengdu, China). All of derivatization reagents were purchased from Sinopharm Chemical Reagent Co., Ltd (Chengdu, China). Other chemical reagents supplied by Hangjia Chemical Co., Ltd. (Chengdu, China) were all in analytical level.

\section{Instruments and Equipments}

Analysis was carried out on a Shimadzu series liquid chromatography system, equipped with LC-20AT pump and SPDM20A photodiode array detector (both from Shimadzu, Kyoto, Japan), a model OR-2090 optical rotation detector (JASCO, Kyoto, Japan) and an HCT-360 LC column cooler/heater (Au- 
tomatic Science, Tianjin, China). Chromatographic parameters such as peak area, retention time, theoretical plate, etc. were calculated using the Class-VP workstation (Shimadzu, Kyoto, Japan).

\section{Derivatization reaction process for mandelic acid derivatives}

In order to further investigate the influence of spatial barrier and group interaction on separation result, mandelic acid was selected as model molecular which has been successful separated by all kinds of CSP columns, and a series of its derivatives were prepared as following procedure: $0.15 \mathrm{~g}$ mandelic acid was dissolved in $60 \mathrm{ml}$ anhydrous methylene chloride, and then $0.4 \mathrm{~g}$ dicyclimide (DCC) was added as catalyst. This mixture reacted at room temperature for $5 \mathrm{~min}$, and then $1 \mathrm{mmol}$ amine (aniline, methyl aniline, naphthylamine or phenylethylamine) was added and reacted $3 \mathrm{~h}$ under stirring at $30{ }^{\circ} \mathrm{C}$. At the end of the reaction, the crude products were washed with $40 \mathrm{~mL} 1 \mathrm{~mol}$ $\mathrm{mL}^{-1}$ hydrochloride acid solution, sodium hydroxide solution and redistilled water, successively and dried by anhydrous sodium sulfate. Residual solvents were removed under vacuum.

By comparison of separation results for different derivatives, the influence by derivatives structure for separation and related mechanism could be investigated.

\section{Chromatographic Conditions}

The separation of above four kinds of mandelic acid derivatives and prasugrel enantiomers was carried out on different CSPs with hexane-alcohol system as the mobile phase, the detection wavelength was $254 \mathrm{~nm}$, flow rate was $1.0 \mathrm{~mL} \cdot \mathrm{min}^{-1}$ and column temperature was $25^{\circ} \mathrm{C}$.

\section{Results and discussion}

\section{Enantioseparation Research of Mandelic Acid Derivatives on CSPs}

From the resolution results shown in Table 3, it is obvious Whelk-O1 should be the most optimal CSP (relative chromato-

grams also given in Fig. 1). Moreover, Fig. 1 also shows the resolution of phenylethylamine derivatives is best on WhelkO1 chiral column under same analytical conditions. The difference of separation results about aniline and methyl aniline derivatives is not significant, and the resolution of naphthylamine derivatives is worst. For the structures of four kinds of derivatives, the most obvious difference lies in the steric hindrance around chiral center. The order of steric size is phenylethylamine derivatives $<$ aniline derivatives $\approx p$-aminotoluene derivatives $<$ naphthylamine derivatives, which is also proved by the data in Table 3. The smaller spatial barrier the derivatives provided, the longer retention time and the better resolution could be achieved. In addition, because of the existence of an electron donating $-\mathrm{CH}_{3}$ on benzene ring in $p$-aminotoluene derivatives, the benzene ring becomes $\pi$-base ( $\pi$-donor) group and the interaction force between the analytes and $\pi$-acid ( $\pi$-acceptor) benzene ring of stationary phase is stronger. Therefore their retention time was longer than aniline derivatives, but the separation effect had not obvious difference.

\section{Thermodynamic study on resolution of enantiomers}

Temperature is an important factor in controlling enantiomeric recognition process. In many cases, low column temperature is beneficial to improve resolution, and the chiral separation is mainly an enthalpy-driven process [22]. In the process of chromatographic separation of optical isomers, the relationship of retention factor of solute $(k)$ and column temperature, as well as correlation of separation factor $(\alpha)$ of enantiomers and column temperature can be expressed by Van't Hoff equation and Gibbs-Holmholtz equation as follows:

$$
\begin{gathered}
\ln k=-\frac{\Delta H}{R T}+\frac{\Delta S}{R}+\ln \varphi \\
\ln a=-\frac{\Delta(\Delta G)}{R T}=-\frac{\Delta(\Delta H)}{R T}+\frac{\Delta(\Delta S)}{R}
\end{gathered}
$$

where $\Delta H$ and $\Delta S$ are the enthalpy change and entropy change in retention process of entiomers; $R$ is the gas constant; $T$ is the column temperature (in Kelvin, $\mathrm{K}$ ); $\varphi$ is the phase ratio. Van't Hoff's plots were drawn for logarithm of retention factor (ln $k$ ) versus the reciprocal value of temperature $(1 / T)$ for the

(c)

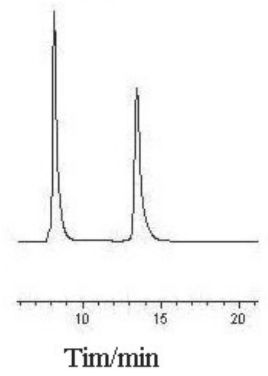

(d)

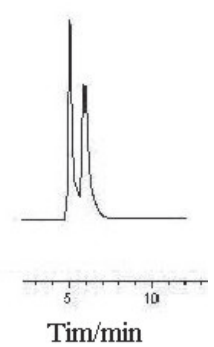

Fig. 1. Chromatograms of (a) aniline derivatives, (b) phenethylamine derivatives, (c) $p$-aminotoluene derivatives and (d) naphthylamine derivatives of mandelic acid on Whelk-O1 chiral column. 
Table 3. Resolution $(R)$ obtained using various columns for four derivatives.

\begin{tabular}{lccccc}
\hline \multirow{2}{*}{ Derivatives } & \multicolumn{2}{c}{ CHI-DMB (Hexane: Isopropyl alcohol = 90:10) } & \multicolumn{3}{c}{ DNB-Leucine (Hexane: Isopropyl alcohol = 90:10) } \\
\cline { 2 - 6 } & $\mathrm{t}_{\mathrm{R} 1}(\mathrm{~min})$ & $\mathrm{t}_{\mathrm{R} 2}(\mathrm{~min})$ & $\alpha$ & $\mathrm{t}_{\mathrm{R} 1}(\mathrm{~min})$ & $\mathrm{t}_{\mathrm{R} 2}(\mathrm{~min})$ \\
\hline Aniline derivatives & 5.973 & 6.421 & 1.159 & 10.923 & 12.000 \\
Phenethylamine derivatives & 4.661 & 5.312 & 1.430 & 14.837 & 17.568 \\
$p$-aminotoluene derivatives & 5.611 & 6.005 & 1.160 & 11.552 & 12.363 \\
Naphthylamine derivatives & 7.787 & 8.693 & 1.195 & 14.400 & 14.400 \\
& OD-H (Hexane: Ethanol $=85: 15)$ & & Whelk-O1 (Hexane: Ethanol=85:15) & 1.097 \\
Aniline derivatives & 7.349 & 9.024 & 1.399 & 7.776 & 12.683 \\
Phenethylamine derivatives & 6.421 & 8.032 & 1.492 & 8.256 & 14.837 \\
$p$-aminotoluene derivatives & 7.605 & 8.043 & 1.098 & 8.139 & 13.429 \\
Naphthylamine derivatives & 9.856 & 10.507 & 1.097 & 5.781 & 6.080 \\
\hline
\end{tabular}

Note: Resolution is equivalent to $R=2\left(t_{R 2}-t_{R 1}\right) /\left(W_{1}+W_{2}\right)$, Where $R=$ resolution, $t_{R 1}=$ retention time of the first peak, $t_{R 2}=$ retention time of the second peak, $W_{1}$ = peak width of first peak, $W_{2}$ = peak width of second peak.

two enantiomers, which yielded the two corresponding straight lines and related regression equations. $\Delta(\Delta H)$ and $\Delta(\Delta S)$ stand for difference of enthalpy change and entropy change of optical isomers, respectively. Among them $\Delta(\Delta S)$ reflects the different of confusion degree change of optical isomers in two phases, and $\Delta(\Delta H)$ presents the difference of interaction force between a couple of optical isomers and chiral stationary phase in different temperature. Moreover, $\alpha$ stands for selectivity factor (the ratio of capacity factors of two isomers, which are $k_{1}$ and $k_{2}$ ). The change in free energy $(\Delta(\Delta G))$ accompanying the separation of two enantiomers was given by [23]:

$$
\Delta(\Delta G)=\Delta(\Delta H)-T \Delta(\Delta S)
$$

With $\ln \alpha$ as ordinate and 1/T as abscissa, Gibbs-Holmholtz curves can be obtained, and related regression equations are shown in Table 4. Through the further calculation, thermodynamic data were obtained (see Table 5).

Results in Table 4 and Table 5 show that there exists linear relationship between $\ln a, \ln k_{1}, \ln k_{2}$ and $1 / T$. $\ln k_{1}$ and $\ln k_{2}$ decrease as the column temperature rises, which means the interaction between CSP and enantiomers is exothermic in nature and the enantioseparation is enthalpy dominated according to the previous studies of enantioseparation [24, 25]. The Van't Hoff curve is basically linear, and the selectivity factor $(\alpha)$ is also a straight line $\left(\mathrm{R}^{2}>0.98\right)$, which shows enantioselective interactions and retention mechanism of the enantiomers on chiral stationary phase do not change obviously with chromatographic separation temperature. Moreover, $\Delta(\Delta H)$ and $\Delta(\Delta S)$ of the two enantiomers of four derivatives are all found as negative values in the process of chromatographic retention, which also indicates the enantioseparation of derivatives of mandelic acid on Whelk-O1 column is a chromatographic process under enthalpy control. Contrarily, the enantioselective interactions are entropic-driven in nature [26]. In addition, the negative entropy change is not benefit for the occurrence of the resolution process, which must be compensated by the enthalpy released in chiral recognition to ensure successful separation of the enantiomers.

\section{Analysis for resolution mechanism}

"Brush" type CSPs are composed by chiral selector, spacer arm and solid support, and the chiral selector usually has $\pi$ donor groups, $\pi$-acceptor groups or the groups that could form multiple hydrogen bonds. The chiral recognition provided by

Table 4. Regression relativity of derivatives of mandelic acid.

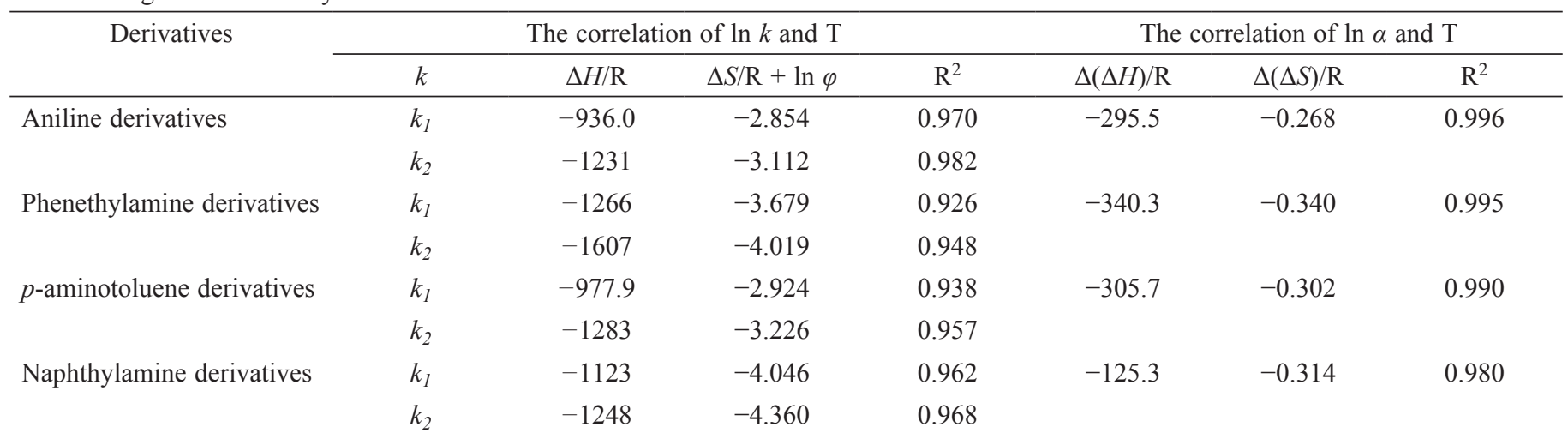


Table 5. The thermodynamic parameters of the enantioseparation of derivatives of mandelic acid on Whelk-O1 column.

\begin{tabular}{lccccccc}
\hline \multicolumn{1}{c}{ Derivatives } & $\Delta \mathrm{H}_{1}$ & $\Delta \mathrm{H}_{2}$ & $-\Delta(\Delta \mathrm{H})$ & $-\Delta(\Delta \mathrm{S})$ & $-\Delta(\Delta \mathrm{G})$ & $\mathrm{T}_{\mathrm{hm}} * / \mathrm{K}$ & $\mathrm{T}_{\beta} * * / \mathrm{K}$ \\
\hline Aniline derivatives & -7.781 & -10.234 & 2.456 & 2.228 & 1.792 & 298.15 & 1102.334 \\
Phenethylamine derivatives & -10.525 & -13.360 & 2.829 & 2.827 & 1.986 & 298.15 & 1000.707 \\
$p$-aminotoluene derivatives & -8.130 & -10.666 & 2.541 & 2.511 & 1.792 & 298.15 \\
Naphthylamine derivatives & -9.336 & -10.375 & 1.041 & 2.611 & 0.263 & 298.15 & 398.698 \\
\hline
\end{tabular}

* $\mathrm{T}_{\mathrm{hm}}$ is the mean harmonic temperature in Van't Hoff analysis [27].

$* * \mathrm{~T}_{\beta}$ is the isokinetic temperature [28].

these groups can meet the requirements of three-point interaction principle [26]. Whelk-O1 chiral chromatographic column is based on the silica gel matrix bonded with chiral selector of naproxen, which is the most broadly applicable of the $\pi$-association CSPs. In the stereoselective separation with this kind of CSP, the major interaction usually occurs in the following ways:

a) $\pi-\pi$ action between the aromatic rings of the enantiomers and CSP;

b) hydrogen bond action between the secondary amine or carbonyl groups on the CSP and acidic proton, hydroxyl or amine groups on the enantiomers;

c) dipole-dipole action;

d) space-steric effect of nonpolar group when it is close to the CSP chiral center.

Potential interaction points between mandelic acid derivatives and Whelk-O1 stationary phase in this study is depicted in Fig. 2. It can be seen more vividly that various substituent groups would lead to different steric hindrance around chiral carbon atom, which could influence the strength of hydrogen bond between the enantiomer and stationary phase. Difference of intermolecular force is the basis of successful separation for two enantiomers. According to Table 5, difference of enthalpy change of the four derivatives is $2.456 \mathrm{~kJ} \mathrm{~mol}^{-1}, 2.829$ $\mathrm{kJ} \mathrm{mol}^{-1}, 2.541 \mathrm{~kJ} \mathrm{~mol}^{-1}, 1.041 \mathrm{~kJ} \mathrm{~mol}^{-1}$, respectively, which are all relatively small. It suggests that the difference of chiral recognition is small under the combinational effect of above four types of intermolecular interactions. Absolute value of $\Delta(\Delta H)$ of phenethylamine derivative enantiomers is the largest in four kinds of derivatives and their resolution factor is also the

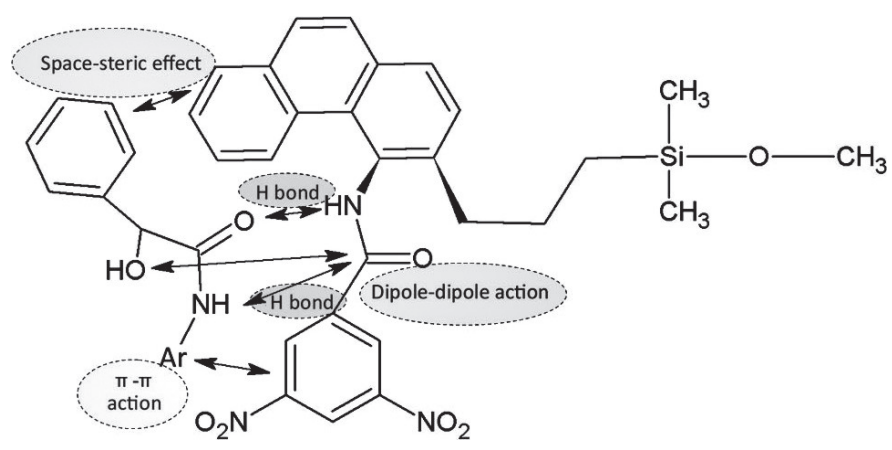

Fig. 2. Various interactions between the derivatives of mandelic acid and Whelk-O1 CSP. largest, which is expected to prove our conclusion about steric hindrance effect and its role in stereoselective separation.

On the basis of calculation of the combination ability with CSP for $R$-enantiomer and $S$-enantiomer, it could help us know key reasons of whether enantiomers can be separated by some kind of CSP. In our theoretical study of thirteen typical chiral analytes (synthomycin, naproxen, ibuprofen, fenoprofen, 2methoxy-N-((R)-1- phenylethyl) acetamide, etc.) docked with Whelk-O1 CSP by AutoDock 4.0 molecular modeling simulation software, most of the docking results agreed with the experimental data [29]. The difference of binding free energy for enantiomers $\left(\Delta \Delta G_{\text {binding }}\right)$ can indicate whether these enantiomers are able to be separated by CSP. The greater the absolute value of $\Delta \Delta G_{\text {binding }}$ is, the easier enantiomers can be separated by CSP. For instance, $R$ - and $S$-enantiomer of fenoprofen both formed $\pi-\pi$ stack and one H-bond with Whelk-O1 in two points, respectively. The largest different energy existed between $\mathrm{H}$ atom in carboxyl group of the enantiomers and Whelk-O1. There were strong interactions only between the $S$-enantiomer and the CSP because of the different configurations, which was eluted later than $R$-enantiomer in LC chromatogram. Many application cases have shown that the computational chemistry methods are convenient not only for predict separation effect and elution sequence, but also for researchers to explore detailed mechanism of interactions between the enantiomers and CSP [30].

\section{Enantioseparation Research of Prasugrel on five CSPs}

Prasugrel, chemical called 5-((1S)-2- cyclopropyl-1-(2-fluorophenyl)-2-oxoethyl)-4H, 5H, 6H, 7H-thieno (3,2-c) pyridin2 -yl acetate, is a kind of new oral effective thienopyridine drug. As a member of the thienopyridine class of Adenosine diphosphate (ADP) receptor inhibitors, US Food and Drug Administration (FDA) has approved its use for the reduction of thrombotic cardiovascular events (including stent thrombosis). Prasugrel contains a chiral centre and thus, exists as two individual enantiomers, which are similar in activity and also can racemize rapidly; therefore, prasugrel is used in clinical with its racemic form [31].

After the experiments about the separation of two enantiomers of prasugrel on the above five chiral stationary phases, it was found that only Chiralcel OD-H column had the trend of resolution. As shown in Fig. 3, while the other columns were unable to achieve obvious separation. After the optimization for 


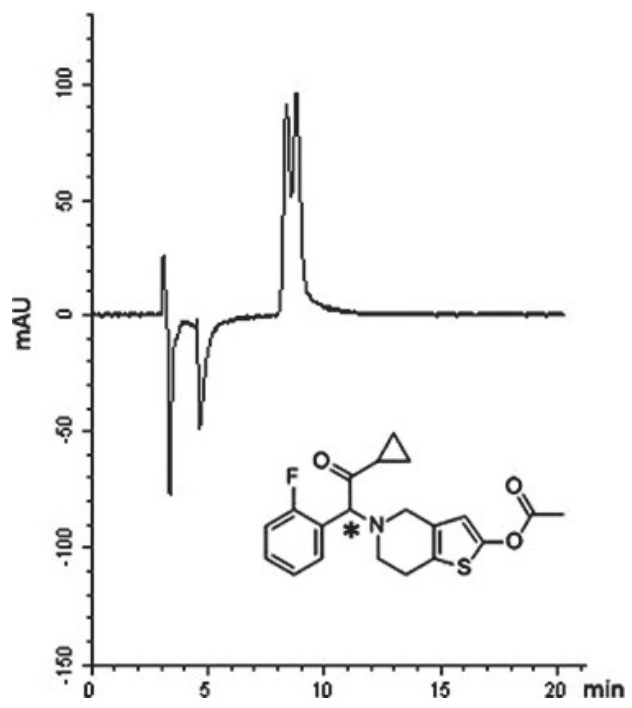

Fig. 3. The structure of prasugrel and chromatogram of its enantiomers (Chiral column: Chiralcel OD-H; mobile phase: $n$-hexane/isopropanol $=90 / 10, \mathrm{~V} / \mathrm{V}$; flow rate: $1 \mathrm{ml} \cdot \mathrm{min}^{-1}$ ).

the polarity and velocity of mobile phase and other chromatographic conditions, the perfect baseline separation of prasugrel enantiomers still could not be obtained on Chiralcel OD-H column. In terms of the structure of prasugrel, all of the three groups adjacent to the chiral center are comparatively large and could result in high steric hindrance and binding free energy, which could block the formation of a stereoselective complex through hydrogen bonding, $\pi-\pi$, dipole-dipole and hydrophobic effects, etc [32]. It is not benefit to the effective formation of these interactions between CSPs and analytes, and three-point contact model discovers that the interactions are needed to be strong enough to promote the formation of at least one of two possible diastereomeric associates and then result in successful resolution [33]. For the example of prasugrel, the weak interactions and chiral recognition lead to incomplete separation. Moreover, for those successfully separated enantiomers in Table 2, the steric hindrance of those groups of adjacent to chiral center in their structures was all smaller than that of prasugrel. Therefore, the important conclusion could be drawn as: the appropriate volume of chiral center adjacent groups and their distance from chiral center are key factors for successful chiral resolution, although the contribution of steric hindrance to the steroselectivity mechanism is easy to be underestimated in those published studies and the effect of spatial barrier is always accompanied with that of crucial group interaction on chiral recognition [32]. The effective localization of the analytes within the CSP active region is necessary, which could be investigated by the experiments and mimic docking.

\section{Conclusion}

In this paper, two important separation aspects-stationary phase type and enantiomer structure are discussed on the basis of the experimental data. Polysaccharide derivative CSP separation range can provide the broadest scope of application in our experiment, and various polysaccharide-type CSPs possess good complementarity. Thus in the selection of chiral stationary phase, a priority selection can be given to this class of stationary phases for chiral separation. Moreover, enantiomers structure is another important factor to influence the chiral recognition, and intermolecular forces were discussed with emphasis on the steric effect provided by chiral center adjacent groups. Thermodynamic mechanism was also investigated preliminarily. Theoretical study with computational chemistry methods is helpful to understand those atoms (and/or group) interactions between enantiomers and CSPs. With the accumulation of more and more further researches, successful separation and prediction are believed to be not headachy work any more for the people in this field.

\section{Acknowledgements}

This work was supported by The National Science Foundation of China (No. 81373284, 81102344) and the Bureau of Science and Technology of Mianyang City of China (No.10Y003-8).

\section{References}

1. Zhang, T.; Nguyen, D.; Franco, P. J. Chromatogr. A. 2008, 1191, 214-222.

2. Luo, J.; Wang, Q. Y.; Zhang, Y. W.; Song, H.; Yao, S. American Lab. 2011, 43, 11-15.

3. Zhang, Y. W.; Yao, S.; Zeng, H.; Song, H. Curr. Pharm. Anal. 2010, 6, 114-130.

4. Beesley, T. E. LC GC Europe. 2011, 24, 270-276.

5. Pirkle, W. H.; House, D. W.; Finn, J. M. J. Chromatogr. 1980, 192, 143-158.

6. Sun, J.; Lan, X. Q.; Tang, X. H.; Xu, X.; Song, H. Chin. J. Anal. Chem. 2006, 34, 158-160.

7. Tang, Q.; Chen, X. Y.; Song, H. Chin. J. Anal. Chem. 2007, 35, 240-243.

8. Liang, Y. M.; Song, H.; Fu, C.; Zhen, W. L. Chin. J. Anal. Chem. 2003, 31, 1253-1255.

9. Tang, Q.; Song, H.; Fu, C.; Lei, Y. C.; Chen, X. Y. Chin. J. Anal. Chem. 2004, 32, 755-758.

10. Zhen, W. L.; Song, H.; Liang, Y. M.; Fu, C. Chin. J. Chromatogr. 2003, 21, 239-241.

11. Xu, X.; Song, H.; Sun, J.; Fu, C.; Shen, S. J. Chin. J. Anal. Lab. 2007, 26, 89-92.

12. Zhang, Y.W.; Yang, D.; Yao, S.; Cao, Y.; Song, H. J. Chromatogr. Sci. 2011, 49, 67-71.

13. Zhang, Y. W.; Song, H.; Lin, F.; Lv, G..; Yao, S. Chinese J. Instrumental Anal. 2010, 29, 739-742.

14. Du, T. T.; Li, L. J.; Song, H.; Zhang, Y. W.; Wang, X.; Luo, J. Chin. J. Anal. Lab. 2009, 28, 76-79.

15. Zhang, Y. W.; Yang, D.; Xie, Y. M.; Liu, Q. S.; Wang, X.; Song, H. Chinese J. Anal. Lab. 2009, 28, 72-75.

16. Li, J.F.; Du, T. T.; Lan, X.Q.; Zhang, Y. W.; Song, H. Chinese J. Anal. Chem. 2007, 35, 1195-1198.

17. Tang, X. H.; Yang, D.; Xie, Y. M.; Cao, Y.; Song, H. Chromatogr. 2009, 70, 365-369. 
18. Xie, Y. M.; Luo, J.; Tang, X. H.; Yang, D.; Huo, X.F.; Liu, A. Chromatogr. 2009, 69, 1025-1029.

19. Zhang, Y. W.; Luo, J.; Zeng, H.; Yao S.; Chen, H.; Song H. Asian J. Chem. 2013, 25, 1353-1356.

20. Wang, Q. Y.; Zhang, Y. W.; Yang, D.; Wang, Y. M.; Song, H. J. China Pharm. 2009, 31, 2456-2458.

21. Francotte, E. J. Chromatogr. 1994, 666, 565-601.

22. Weng, W.; Yao, B. X.; Cheng, X. Q.; Cheng, Y. Z.; Lin, W. S.; Zeng, Q. L. Prog. Chem. 2006, 18, 1056-1064.

23. Berthod, A.; He, L.; Beesley, T. E. J. Chromatogr. A. 2004, 1060, 205-214.

24. O'Brien, T.; Crocker, L.; Thompson, T. R. K.; Toma, P. H.; Conlon, D. A.; Feibush, B.; Moeder, C.; Bicker, G.; Grinberg, N. Anal. Chem. 1997, 69, 1999-2007.

25. Ma, S.; Tsui, H. W.; Spinelli, E.; Busacca, C. A.; Franses, E. I.; Wang, N. H. L.; Wu, L.; Lee, H., Senanayake, C.; Yee, N.; Gonella, N.; Fandrick, K., Grinberg, N. J. Chromatogr. A. 2014, 1362, 119-128.
26. Pirkle, W. H.; Burke, J. A. J. Chromatogr. 1991, 557, 173185.

27. Krug, R. R.; Hunter, W. G.; Grieger, R. A. J. Phys. Chem. 1976, 80, 2341-2351.

28. McMinn, W. A. M.; Al-Muhtaseb, A.H.; Magge, T. R. A. Food Res. Int. 2005, 35, 505-510.

29. Cao, Y.; Wang, X. L.; Yao, S.; Cheng, Q.; Song, H. J. Chem. Soc. Pakistan. 2012, 34, 102-106.

30. Lämmerhofer, M. J. Chromatogr. A. 2010, 1217, 814-856.

31. Daniel, J. C.; Emilio, J. E.; David, G.; Mark, S. S.; Andreas, J. G.; Maheshbhai, R. D.; James, R. W.; Deborah, J. L. J. Urology. 2014, 192, 1026-1034.

32. Berthod, A. Chiral Recognition in Separation Methods, SpringerVerlag, Berlin, 2010.

33. Berthod, A. Anal. Chem. 2006, 78, 2093-2099.

34. Hassan, Y. A.; Imran, A. Chiral Separations by Liquid Chromatography and Related Technologies, Marcel Dekker Inc., New York, 2003. 